\title{
PHASE FLUCTUATIONS AND BCS-LP CROSSOVER IN 2D SHORT COHERENCE LENGTH SUPERCONDUCTORS
}

\author{
B. TobIJaszewska and R. Micnas \\ Institute of Physics, A. Mickiewicz University \\ Umultowska 85, 61-614 Poznań, Poland
}

We study the effects of phase fluctuations in $2 D$ short-coherence length superconductors on the basis of the extended Hubbard model with intersite attraction. In the Kosterlitz-Thouless scenario the critical temperatures for anisotropic pairings were determined and compared with the ones from BCS Hartree-Fock approximation. The Uemura-type plots, i.e. the critical temperature vs. zero temperature phase stiffness, were obtained for extended $s$-wave and $d_{x^{2}-y^{2}}$-wave pairings. We also discuss the crossover from BCS to local pair superconductivity for $d$-wave pairing.

PACS numbers: 74.20.-z, 71.10.Fd, 74.20.Fg, 74.20.Mn, 74.72.-h

\section{Introduction}

Several experiments point out that the pairing in hole doped cuprate high temperature superconductors (HTS) is characterized by a strong $d$-wave component [1]. Moreover, due to short coherence length and small superfluid density in the underdoped regime, one also expects that the phase fluctuations are important and can have profound effect on the HTS properties [2]. One of the simplest models of short coherence length superconductor, which can account for both $s$ - and $d$-wave symmetry, is the extended Hubbard Hamiltonian with intersite attraction

$$
H=\sum_{i j \sigma}\left(t_{i j}-\mu \delta_{i j}\right) c_{i \sigma}^{\dagger} c_{j \sigma}+U \sum_{i} n_{i \uparrow} n_{i \downarrow}+\frac{1}{2} \sum_{i j \sigma \sigma^{\prime}} W_{i j} n_{i \sigma} n_{j \sigma^{\prime}}
$$

where $t_{i j}$ is the transfer integral, $U$ is the on-site and $W_{i j}$ is the intersite interaction, and $\mu$ is the chemical potential. We will analyze the superconducting properties of (1) in the case of two-dimensional square lattice with $W<0$, i.e. the intersite near-neighbour (nn) attraction. The self-consistent equations for the superconducting order parameter and the free energy are obtained in the broken symmetry Hartree-Fock (HF)-BCS approximation for Hamiltonian (1) [3]. One gets

$$
\Delta_{k}=\frac{1}{N} \sum_{q}\left(-U-W_{k-q}\right) \Delta_{q} F_{q}, \quad F_{q}=\frac{1}{2 E_{q}} \tanh \left(\frac{\beta E_{q}}{2}\right),
$$


where $W_{\boldsymbol{k}}$ is the Fourier transform of $W_{i, j}$ and $\beta=1 / k_{\mathrm{B}} T$. The quasiparticle energy is given by $E_{q}=\sqrt{\bar{\epsilon}_{\boldsymbol{q}}^{2}+\left|\Delta_{q}\right|^{2}}, \bar{\epsilon}_{q}=\epsilon_{q}-\bar{\mu}$, where the electron dispersion is $\epsilon_{q}=-2 t\left[\cos \left(q_{x} a\right)+\cos \left(q_{y} a\right)\right]-4 t_{2} \cos \left(q_{x} a\right) \cos \left(q_{y} a\right)$ for the next nearest neighbour (nnn) hopping $\left(t_{2}\right)$, and $\bar{\mu}=\mu-n(U / 2+4 W)$. We consider the singlet pairing and the gap function takes the form: $\Delta_{k}=\Delta_{0}+\Delta_{\gamma} \gamma_{k}+\Delta_{\eta} \eta_{k}$, where $\gamma_{\boldsymbol{k}}=$ $2\left[\cos \left(k_{x} a\right)+\cos \left(k_{y} a\right)\right]$ and $\eta_{k}=2\left[\cos \left(k_{x} a\right)-\cos \left(k_{y} a\right)\right]$. The first and second terms refer to the on-site and extended $s$-wave $\left(s^{*}\right)$ and the third to the $d_{x^{2}-y^{2} \text {-wave }}$ pairing. The resulting equations for the gap amplitudes have to be solved together with the equation determining the chemical potential $\bar{\mu}$ :

$$
n-1=-\frac{2}{N} \sum_{\boldsymbol{q}} \bar{\epsilon}_{\boldsymbol{q}} F_{q}
$$

The density of states (DOS) is given by $N(\omega)=\frac{1}{N} \sum_{k}\left[u_{k}^{2} \delta\left(\omega-E_{k}\right)+\right.$ $\left.\left|v_{\boldsymbol{k}}\right|^{2} \delta\left(\omega+E_{\boldsymbol{k}}\right)\right], u_{\boldsymbol{k}}^{2}=\left(1+\bar{\epsilon}_{\boldsymbol{k}} / E_{k}\right) / 2, u_{\boldsymbol{k}}^{2}+\left|v_{\boldsymbol{k}}\right|^{2}=1$, and the momentum distribution at $T=0 \mathrm{~K}$ is $n_{k}=\left|v_{k}\right|^{2}$. The HF transition temperature is the one at which the gap amplitude vanishes and gives the pair-breaking temperature. When the phase fluctuations of the order parameter are taken into account, the phase transition generally occurs at a temperature lower than that given by HFA. We assume that in 2D this temperature can be determined within the Kosterlitz-Thouless (KT) scenario [4] and consequently we have estimated it from the relation for the universal jump of the superfluid stiffness $\rho_{s}$ at $T_{\mathrm{c}}[4,5]$ :

$$
\frac{2}{\pi} T_{\mathrm{c}}=\rho_{s}^{-}\left(T_{\mathrm{c}}\right)
$$

The superfluid stiffness $\rho_{s}$, which is proportional to $1 / \lambda^{2}\left(\lambda^{-2}=\left(16 \pi e^{2} / \hbar^{2} c^{2}\right) \rho_{s}, \lambda\right.$ is the London penetration depth) [5], can be obtained from the linear response theory [6] and is given by

$$
\rho_{s}^{\alpha}=\frac{1}{2 N} \sum_{\boldsymbol{k}}\left\{\left(\frac{\partial \epsilon_{\boldsymbol{k}}}{\partial k_{\alpha}}\right)^{2} \frac{\partial f\left(E_{\boldsymbol{k}}\right)}{\partial E_{\boldsymbol{k}}}+\frac{1}{2} \frac{\partial^{2} \epsilon_{\boldsymbol{k}}}{\partial k_{\alpha}^{2}}\left[1-\frac{\bar{\epsilon}_{\boldsymbol{k}}}{E_{k}} \tanh \left(\frac{\beta E_{\boldsymbol{k}}}{2}\right)\right]\right\},
$$

where $f\left(E_{k}\right)$ is the Fermi-Dirac distribution function and $\alpha=x, y, z$.

\section{Results}

By numerical solution of the above equations we have determined the superfluid stiffness, the critical temperatures and constructed the plots of $T_{\mathrm{c}}$ vs. $1 / \lambda^{2}(0)$ (the Uemura plots) in BCS-HFA and KT scenario (Fig. 1). In Fig. 1a we present the Uemura plot for $n n$ hopping and $|W / 4 t|=0.5$. The curves for $s$-wave KT temperatures have the form similar to those obtained experimentally by Uemura [7], especially the linear fragment. For small $n$, the points follow the universal line $\pi \rho_{s}(0) / 2$ and the KT temperatures are well estimated by the zero-temperature value of superfluid stiffness. Indeed, the $\mathrm{HF}$ and $\mathrm{KT}$ temperatures differ showing the separation of scales for the pairing and for the phase coherence. Additionally, in the inset of Fig. 1a the order parameters and $\rho_{s}(0)$ vs. $n$ (for $U=0$ ) are shown, which illustrate a separation of the above scales at $T=0 \mathrm{~K}$. For low $n$, and for $s^{*}$-wave symmetry $\rho_{s}(0)$ is smaller than the order parameter in contrast to higher $n$, where this behaviour is reversed in the way characteristic of the weak coupling 
BCS theory. The dotted line shows the relation $\rho_{s}(0)=\hbar^{2} n_{s} / 4 m(=(n / 2) t)$ in a dilute limit, appropriate for continuum fermions $\left(n_{s}=n\right.$, with $n_{s}$ being superfluid carrier density). In general, $n_{s}$ is smaller than $n$. We also point out that the conventional BCS-MF approximation (Fig. 1, empty symbols) cannot account for the Uemura type scaling. For $d_{x^{2}-y^{2}}$-wave pairing the plot has no characteristic Uemura shape for the nn hopping. However, if the nnn hopping is included, a dramatic change in its behaviour is observed. With increasing values of $t_{2}$ (taken with opposite sign to $t$ ) the plot becomes more and more reminiscent of the ones obtained for the $s$-wave pairing (Fig. 1b) and the Uemura scaling is recovered for the $d_{x^{2}-y^{2}}$ pairing symmetry.
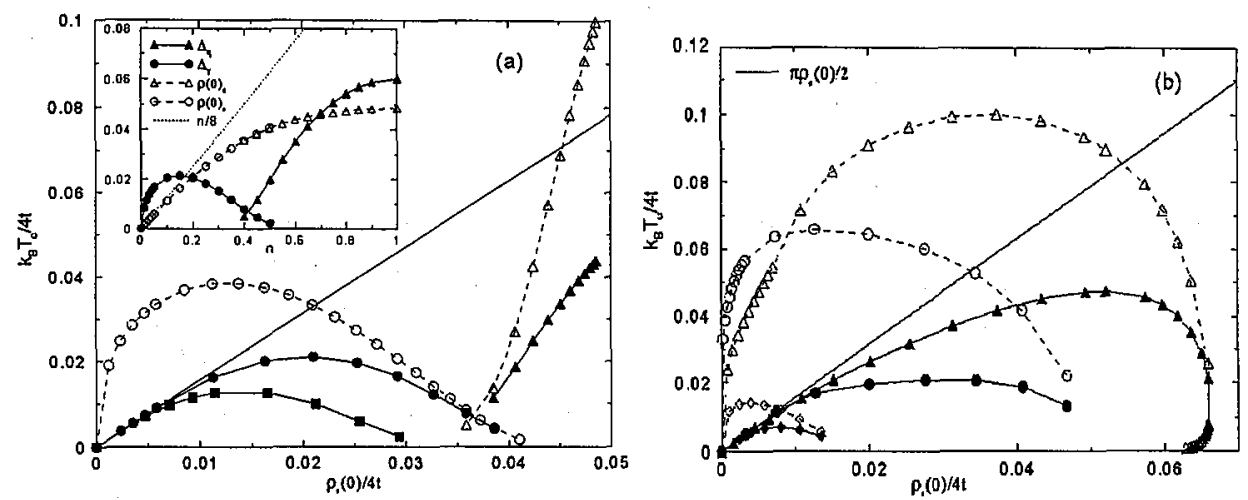

Fig. 1. Uemura-type plots (the critical temperature vs. superfluid stiffness at $T=0),|W / 4 t|=0.5$, controlling parameter $n$. The increasing $\rho_{s}(0)$ corresponds to the increase in $n$. The straight line $\pi \rho_{s}(0) / 2$ is an upper bound on the phase ordering temperature. Part (a) is for $n n$ hopping and part (b) is for the nnn hopping $t_{2}=-0.45 t$. Dashed lines with empty symbols denote HF $T_{c}$, solid lines with filled symbols are for $\mathrm{KT} T_{c}$; triangles $-d$-wave, circles $-s^{*}$-wave $U / 4 t=0$, squares $-s^{*}$-wave $U / 4 t=0.5$, diamonds $-s^{*}$-wave $U / 4 t=1, W_{2} / 4 t=0.35\left(W_{2}\right.$ is the nnn Coulomb repulsion).

We also observe that when the points collapse on the line $\pi \rho_{s}(0) / 2$ for $d$-wave plot in Fig. 1b then the crossover from BCS to preformed pairs behaviour can take place. The crossover line can be determined from the condition that the renormalized chemical potential $\bar{\mu}$ falls below the band bottom $\epsilon_{0}$. This is associated with a sharp change in the gap to single particle excitations for a pure $d$-wave pairing, which for $\bar{\mu} \leq \epsilon_{0}$ changes to the nodeless gap. In Fig. 2 we give the boundary values of $n$ as a function of $|W|$ for different ratios of $t_{2} / t$ and $T=0 \mathrm{~K}$. For $|W| / 4 t=0.5$ and $t_{2} / t=-0.45$ the critical value of $n$ is about 0.13 ; for higher $n$ we have BCS pairs, and for lower $n-$ local $d$-wave pairs. To analyze this phenomenon better, in Fig. 3 we show the superconducting DOS for $n$ below and above the critical value. In the BCS regime we have a typical $d$-wave shape with the order parameter amplitude determined by the position of the logarithmic singularity. If $n$ is below the critical value (local pair (LP) regime), then the true gap to single particle excitations opens. In such a case, the gap value is determined 


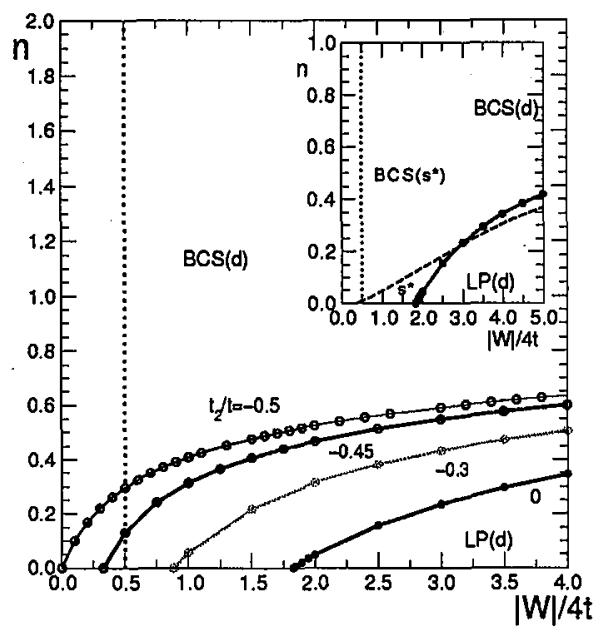

Fig. 2. Lines for which $\bar{\mu}$ falls below the band bottom in the superconducting ground state of $d_{x^{2}-y^{2}}$-symmetry, for different values of the nnn hopping. Inset shows BCS-LP crossover diagram at $T=0 \mathrm{~K}$ for $\mathrm{nn}$ hopping. Dashed line is for $s^{*}$-symmetry $(U=0)$. Below dotted line, which is for $s^{*}$-wave and $U=\infty$ and $n n$ hopping, the $s^{*}$-pairs cannot be bound.
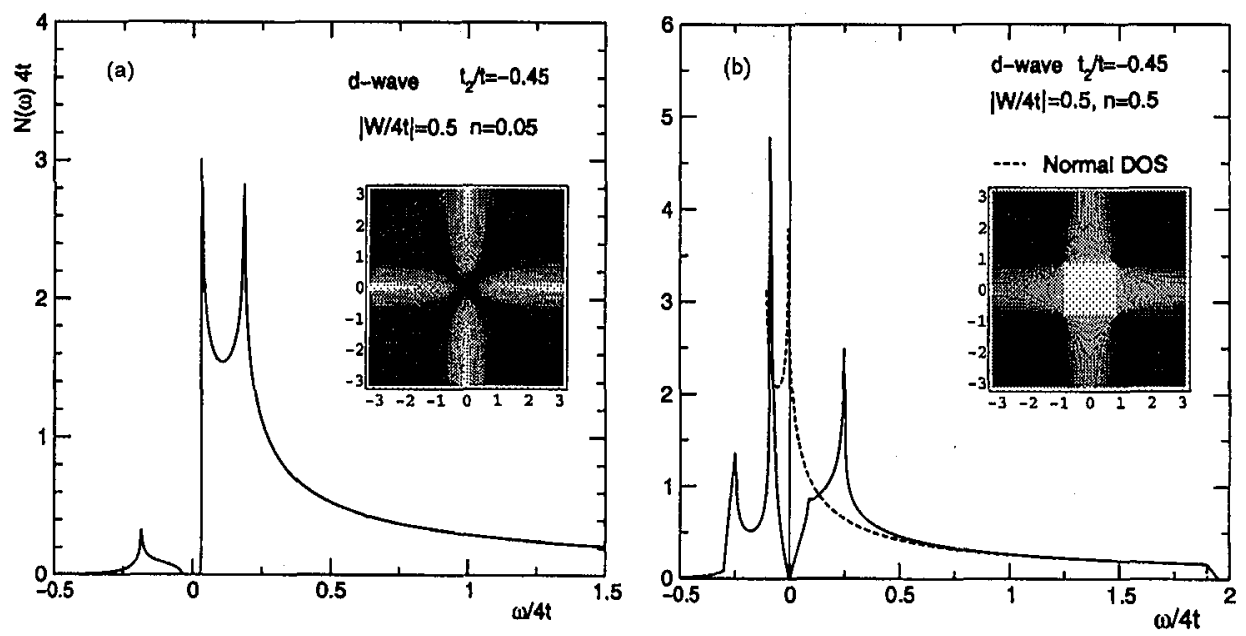

Fig. 3. Superconducting DOS vs. $\omega$ for the density driven crossover in the case of $d$-wave pairing $(T=0 \mathrm{~K})$. Inset plots show the momentum distribution for $d_{x^{2}-y^{2}}$ pairs in the Brillouin zone; part (a) is in the regime of small $n$ - LP case, (b) for high $n$ - BCS case with four nodal points. The brighter the region the larger $n_{k}$. Dotted line shows the DOS in normal state.

by $\left|\bar{\mu}-\epsilon_{0}\right|$, and this gap is nodeless in contrast to the order parameter. Insets in Fig. 3 show the change in the momentum distribution for $d$-wave pairing. It also appears that, in contrast to the $s$-wave pairing, for $d$-wave symmetry at $T=0 \mathrm{~K}$ $\bar{\mu}(n,|W|)$ and $\Delta_{\eta}(n,|W|)$ exhibit a weak singularity at the crossover point. 
To summarize we have obtained the Uemura-type plots for $s^{*}$ and $d_{x^{2}-y^{2}}$-wave pairings, and observe a strong influence of nnn hopping on the $d$-wave Uemura plots. The universal behaviour $T_{\mathrm{c}} \sim \rho_{s}(0)$ is the result of phase fluctations and proximity to the band insulator, which in our case is the $n=0$ point. For an appropriate strength of intersite attraction and the density this is also connected with the crossover from the BCS to the LP superconductivity. In the case of a pure $d$-wave pairing, such crossover is reflected by significant changes in several superconducting characteristics, including the DOS behaviour. A more extended analysis of phase fluctuations and BCS-LP crossover, incorporating also $s+d$ mixing, will be published elsewhere.

\section{Acknowledgments}

This paper was supported by the Committee for Scientific Research grant No. 2 P03B 056 14. R.M. also acknowledges support from Polonium exchange program. We would like to thank S. Robaszkiewicz for useful discussions.

\section{References}

[1] J. Annett, N. Goldenfeld, A.J. Leggett, in: Physical Properties of High Temperature Superconductors, Vol. 5, Ed. D.M. Ginsberg, World Scientific, Singapore 1996, p. 27.

[2] R. Micnas, S. Robaszkiewicz, in: High-Tc Superconductivity 1996: Ten Years after the Discovery, Eds. E. Kaldis, E. Liarokapis, K.A. Müller, NATO ASI Series E, Vol. 343, Kluwer Academic Publishers, The Netherlands 1997, p. 31 and references therein.

[3] R. Micnas, J. Ranninger, S. Robaszkiewicz, S. Tabor, Phys. Rev. B 37, 9410 (1988); R. Micnas, J. Ranninger, S. Robaszkiewicz, ibid., 39, 11653 (1989).

[4] J.M. Kosterlitz, D.J. Thouless, J. Phys. C, Solid State Phys. 6, 1181 (1973).

[5] P.J.H. Denteneer, Guozhong An, J.M.J. van Leeuwen, Phys. Rev. B 47, 6256 (1993); J.M.J. van Leeuwen, M.S.L. du Croo de Jongh, P.J.H. Denteneer, J. Phys. A 29, 41 (1996).

[6] D.J. Scalapino, S.R. White, S. Zhang, Phys. Rev. B 47, 7995 (1993).

[7] Y.J. Uemura, L.P. Le, G.M. Luke, B.J. Sternlieb, W.D. Wu, J.H. Brewer, T.M. Riseman, C.L. Seaman, M.B. Maple, M. Ishikawa, D.G. Hinks, J.D. Jorgensen, G. Saito, H. Yamochi, Phys. Rev. Lett. 66, 2665 (1991); Y.J. Uemura, G.M. Luke, B.J. Sternlieb, J.H. Brewer, J.F. Cardan, W.N. Hardy, R. Kadono, J.R. Kempton, R.F. Kiefl, S.R. Kreitzman, P. Mulhern, T.M. Riseman, D.U. Williams, B.X. Yang, S. Uchida, H. Takagi, J. Gopalakrishnan, A.W. Sleight, M.A. Subramanian, C.L. Chien, M.Z. Cieplak, Gang Xiao, V.Y. Lee, B.W. Statt, C.E. Stronach, W.J. Kossler, X.H. Yu, Phys. Rev. Lett. 62, 2317 (1989); Y.J. Uemura, Physica C 282-287, 194 (1997). 